\title{
Ga13 loss promotes tumor progression in the KPC transgenic mouse model of advanced pancreatic cancer
}

Mario A. Shields ${ }^{1,2,{ }^{*}}$, Christina Spaulding ${ }^{1,3}$, Mahmoud G. Khalafalla ${ }^{1}$, Thao N.D. Pham ${ }^{1}$, and Hidayatullah G. Munshi, ${ }^{1,2,3, *}$

${ }^{1}$ Department of Medicine, Feinberg School of Medicine, Northwestern University, Chicago, IL, USA

2 The Robert H. Lurie Comprehensive Cancer Center, Chicago, IL, USA

3 Jesse Brown VA Medical Center, Chicago, IL, USA

Running title: Effects of epithelial Ga13 loss in vivo

Keywords: Ga13, KC mouse model, KPC mouse model, human PDAC tumors, E-cadherin, mTOR

The authors have declared that no conflict of interest exists.

*Address correspondence to

Mario A. Shields, Ph.D. or Hidayatullah G. Munshi, M.D.

Department of Medicine

Northwestern University Feinberg School of Medicine

303 E. Superior Ave., Lurie 3-220 (M.A. Shields) or Lurie 3-117 (H.G. Munshi)

Chicago, IL 60611, USA.

Phone: (312) 503-0312; mario.shields@northwestern.edu (M.A. Shields)

Phone: (312) 503-2301; h-munshi@northwestern.edu (H.G. Munshi) 


\section{SUMMARY}

Ga13 transduces signals from G protein-coupled receptors. Ga13 is pro-tumorigenic in epithelial cancer cell lines, which contrasts with its tumor-suppressive function in transgenic mouse models of lymphomas. Here we show that while loss of Ga13 in pancreatic cell lines decreases tumor growth in vivo, Ga13 loss in the Kras-driven (KC) mouse model of pancreatic tumor initiation does not affect tumor development or survival. Instead, Ga13 loss in the Kras/Tp53 (KPC) transgenic mouse model of advanced pancreatic cancer promotes well-differentiated tumors with increased tumor burden and reduced survival. Mechanistically, Ga13 loss in the KPC mouse model enhances E-cadherin-mediated cell-cell junctions and mTOR signaling. Importantly, human pancreatic cancers with low Ga13 expression exhibit increased E-cadherin protein expression and mTOR signaling. This work establishes a context-dependent role of Ga13 in pancreatic tumorigenesis, demonstrating a tumor-suppressive role in transgenic mouse models of advanced pancreatic cancer. 


\section{INTRODUCTION}

Ga proteins transduce signals from G protein-coupled receptors (GPCRs), the largest class of cell surface proteins that regulate a plethora of biological processes (Oldham and Hamm, 2008). Ga proteins are grouped into Gas (s, stimulatory), Gai (i, inhibitory), Gaq, and Ga12 families (Syrovatkina et al., 2016). Mutations in the GNAS gene, which encodes Gas, contribute to the formation of intraductal papillary mucinous neoplasms (IPMNs) (Molin et al., 2013; Wu et al., 2011), precursor lesions of the pancreas that can progress to pancreatic ductal adenocarcinoma (PDAC) tumors (Farrell and Fernandez-del Castillo, 2013; Matthaei et al., 2011). Over $90 \%$ of human IPMNs harbor either KRAS or GNAS mutations or both (Molin et al., 2013; Wu et al., 2011). Co-expression of mutant Gnas and mutant Kras in mouse models of pancreatic tumorigenesis induces IPMN lesions with a well-differentiated phenotype (Ideno et al., 2018; Patra et al., 2018; Taki et al., 2016). Notably, loss of Tp53 in mouse models of IPMN promotes invasive PDAC lesions (Patra et al., 2018).

While the role of Gas in pancreatic cancer is now well recognized, the role of other $G$ proteins in pancreatic tumor initiation and progression has yet to be fully defined. Ga13, a member of the Ga12 family, has been implicated in cell migration downstream of GPCRs and receptor tyrosine kinases (Kelly et al., 2007; Kelly et al., 2006; Kozasa et al., 2011) and has been shown to mediate invasion of human PDAC cells (Chow et al., 2016). These results suggest that Ga13 is pro-tumorigenic in many different cancers, including pancreatic cancer. However, studies in B cell lymphomas have found that Ga13 functions as a tumor suppressor (Healy et al., 2016; Muppidi et al., 2014; O'Hayre et al., 2016). The GNA13 gene is frequently mutated in germinal center-derived B-cell lymphomas, resulting in loss of Ga13 function (Muppidi et al., 2014). Loss of Ga13 in germinal center B cells resulted in the dissemination of these cells and protected them against cell death (Muppidi et al., 2014). Notably, loss of Ga13 in combination with MYC overexpression in germinal center B cells promoted lymphomas (Healy et al., 2016). These results demonstrate that Ga13 functions as a tumor suppressor in 
lymphomas (Healy et al., 2016; Muppidi et al., 2014; O'Hayre et al., 2016), which contrasts with its function in epithelial cancers (Chow et al., 2016; Kelly et al., 2007; Kelly et al., 2006; Kozasa et al., 2011; Rasheed et al., 2018; Zhang et al., 2018).

It is important to note that many of the studies in epithelial cancers, including our studies in pancreatic cancer (Chow et al., 2016), were conducted in transformed cell lines. However, it is not known whether Ga13 is pro-tumorigenic or tumor-suppressive in transgenic mouse models of epithelial cancer. Here, we evaluate the effects of Ga13 loss in transgenic mouse models of pancreatic tumorigenesis.

\section{RESULTS}

Ga13 loss does not affect tumor development in the KC transgenic mouse model. To investigate the contribution of Ga13 to epithelial tumor development and progression in vivo, we evaluated the effects of Ga13 loss in the LSL-Kras ${ }^{\mathrm{G} 12 \mathrm{D}} / \mathrm{Pdx1-Cre} \mathrm{(KC)} \mathrm{transgenic} \mathrm{mouse} \mathrm{model.}$ The KC mouse model utilizes a pancreas-specific Pdx1 promoter-driven Cre recombinase to knock-in the oncogenic mutant Kras ${ }^{\mathrm{G} 12 \mathrm{D}}$ into the endogenous locus (Hingorani et al., 2003). Since the Kras mutation is an early event in pancreatic cancer tumorigenesis (Hingorani et al., 2003), the KC mouse model allows us to evaluate the effects of targeting Ga13 on pancreatic tumor initiation (Pham et al., 2021). KC mice were crossed with mice expressing the floxed allele of Gna13 to generate KCGa13fl/+ and KCGa13fl/fl mice (referred to as KCGfl/+ and KCGfl/fl mice, respectively; Fig. 1A). The KCGfl/+ and KCGfl/fl mice showed decreased pancreatic expression of Ga13 at the mRNA and protein levels (Figs. 1A and 1B). There was no effect on Gna11, Gna12, Gnaq, or Gnas expression in the KCGfl/fl mice compared to $\mathrm{KCG}+/+$ mice (Fig. 1B). We next evaluated the effects of Ga13 loss on the lesion burden, proliferation (Ki67), and apoptosis (cleaved caspase 3) in the KCGfl/+ and KCGfl/fl mice. We found no difference in the lesion burden in the $\mathrm{KCG}+/+, \mathrm{KCGfl} /+$, and $\mathrm{KCGfl} / \mathrm{fl}$ mice at either 3or 6-months of age (Fig. 1C). There was also no difference in cleaved caspase 3 (data not 
shown) or Ki67 staining in the KCGfl/fl mice compared to $\mathrm{KCG}+/+$ mice (Fig. 1D). Finally, we evaluated the effects of Ga13 loss on survival in the KC mouse model. There was no difference in the survival of KC, KCGfl/+, and KCGfl/fl mice (Fig. 1E).

\section{Cell lines established from KC mice with Ga13 loss grow slower in vitro and in vivo.}

Since the previous studies showing Ga13 as pro-tumorigenic were conducted using epithelial cell lines (Chow et al., 2016; Kelly et al., 2007; Kelly et al., 2006; Kozasa et al., 2011; Rasheed et al., 2018; Zhang et al., 2018), we established cell lines from KC, KCGfl/+ and KCGfl/fl mice to evaluate the effects of Ga13 loss. While the KCGfl/+ cell line showed minimal loss of Ga13 protein, the KCGfl/fl cell line showed a near-complete loss of Ga13 (Fig. 2A). The KCGfl/fl cell lines showed decreased ability to grow on 2D surfaces (Fig. 2B) and reduced ability to grow and invade in 3D culture conditions (Fig. 2C). When these cell lines were injected into the flank of $\mathrm{B} 6$ mice, the $\mathrm{KCGfl} /+$ cells grew slower than the $\mathrm{KCG}+/+$ cells (Fig. 2D). Notably, the KCGf//fl cells failed to develop tumors in vivo (Fig. 2E).

Expression of Ga13 shRNA in pancreatic cell lines decreases tumor growth in vivo. To further assess the effects of targeting Ga13 in established cell lines, we generated doxycyclineinducible KC cell lines expressing either shRNA against GFP (control) or Ga13 to (Supplemental Fig. S1A). When these cell lines were injected in the flank or in the pancreas, loss of Ga13 significantly decreased the growth of KC cell lines in vivo (Supplemental Figs. S1B and S1C).

\section{KPC mice with Ga13 loss develop differentiated tumors with increased E-cadherin} expression. We next evaluated the extent to which Ga13 loss affects tumor progression in the KPC mouse model. The KPC mouse model is a well-established model that expresses mutant Kras and mutant p53 in the mouse pancreas (Hingorani et al., 2005a). We generated KPC mice 
with heterozygous loss of Ga13 in the mouse pancreas (referred to as KPCGfl/+; Fig. 3A). The KPCGfl/+ mice showed decreased pancreatic expression of Ga13 (Fig. 3B). At 3 months of age, the KPCGfl/+ developed increased lesion burden compared to the KPCG+/+ mice (Fig. 3C). Notably, at the survival endpoint, the KPCGfl/+ mice developed well-differentiated tumors with increased E-cadherin staining and increased proliferation (Figs. 3D-F).

\section{KPCGfl/+ mice demonstrate increased mTOR signaling and decreased survival. To} understand why the KPCGfl/+ tumors show increased proliferation, we analyzed human data to identify signaling pathways in human PDAC tumors associated with decreased Ga13 expression. We initially evaluated the relationship between E-cadherin and Ga13 in human PDAC tumors using the publicly accessible The Cancer Genome Atlas (TCGA) data in the cBioportal (Supplemental Table S1) (Cerami et al., 2012; Gao et al., 2013). Human PDAC tumors with low Ga13 expression showed increased E-cadherin protein expression, but there was no difference in E-cadherin mRNA levels (Fig. 4A). There was increased expression of $\beta$ catenin (CTNNB1) and claudin-7 (CLDN7) in human tumors with reduced Ga13 expression (Fig. 4A). There was also evidence of increased activation of mTOR signaling, with increased protein expression of RPS6, p-PDK1(S241), EEF2, and MTOR in human PDAC tumors with reduced Ga13 expression (Fig. 4B). Human PDAC tumors with reduced Ga13 expression showed no difference in RPS6 mRNA levels, but a statistically significant reduction in MTOR mRNA levels (Fig. 4B). Notably, while we did not see increased levels of p-PDK1 in tumors developing in the KPCGfl/+ tumors compared to KPC tumors (data not shown), we found that there was increased expression of Rps6 and Mtor in the KPCGfl/+ tumors compared to $\mathrm{KPCG}+/+$ mice (Fig. 4C). Finally, we found significantly reduced survival of KPCGfl/+ mice compared to KPC mice (Fig. 4D). These results suggest a tumor-suppressive role for Ga13 in advanced PDAC tumors. 


\section{DISCUSSION}

GPCRs are targets of several currently approved therapies (Hauser et al., 2017). There is increasing interest in understanding the role of effectors downstream of GPCRs and determining whether these effectors could be potential therapeutic targets (Hauser et al., 2017). Since GPCRs can signal through the G12 family of small G proteins (Kurose, 2003), many studies have evaluated the contribution of Ga12 and Ga13 to physiologic and pathologic processes (Sriram et al., 2020; Syrovatkina and Huang, 2019; Tutunea-Fatan et al., 2020). These studies, which have been primarily performed in epithelial cancer cell lines, showed that Ga13 functions as a tumor promoter (Chow et al., 2016; Kelly et al., 2007; Kelly et al., 2006; Kozasa et al., 2011; Rasheed et al., 2018; Zhang et al., 2018). We also now show that targeting Ga13 in Kras-expressing pancreatic cell lines reduces or blocks tumor growth in vivo. However, targeting Ga13 in the Kras-driven KC transgenic mouse model of tumor initiation did not affect tumor development or survival of these mice. Instead, we have found that targeting Ga13 in the Kras and p53-driven KPC mouse model promoted tumor development and decreased survival, indicating that Ga13 has a tumor-suppressive role in advanced tumors.

Previously published studies in B cell lymphomas have also shown that Ga13 functions as a tumor suppressor (Healy et al., 2016; Muppidi et al., 2014; O'Hayre et al., 2016). The GNA13 gene is frequently mutated in germinal center-derived B-cell lymphomas, resulting in loss of Ga13 function (Muppidi et al., 2014). Loss of Ga13 in germinal center B cells resulted in the dissemination of these cells and protected them against cell death (Muppidi et al., 2014). Loss of Ga13 in combination with MYC overexpression in germinal center B cells promoted lymphomas (Healy et al., 2016). These results in a lymphoma transgenic mouse and our data in the KPC transgenic mouse model demonstrate a tumor-suppressive role of Ga13 in tumor development and progression. 
Notably, Ga13 loss parallels many of the effects of gain-of-function mutations in Gnas in the pancreas. Co-expression of mutant Gnas with mutant Kras promoted the development of IPMNs (Ideno et al., 2018; Patra et al., 2018; Taki et al., 2016). However, the expression of mutant Gnas in Kras-expressing cell lines decreased the number of colonies in soft agar assay, reduced invasion in vitro, reduced tumor growth in vivo, and induced striking epithelial differentiation (Ideno et al., 2018). Similar to the expression of mutant Gnas in cell lines, we have found that loss of Ga13 in Kras-expressing cell lines decreased proliferation in vitro, decreased growth and invasion in 3D collagen and matrigel, and decreased tumor growth in vivo. In contrast, while co-expression of mutant Kras and Gnas was sufficient to induce IPMNs (Ideno et al., 2018; Patra et al., 2018; Taki et al., 2016), we did not see any apparent effects of Ga13 loss on IPMN development in the KC mouse model. However, when we targeted Ga13 in the KPC mouse model, we found that loss of Ga13 resulted in differentiated tumors, similar to the phenotype seen with co-expression of Gnas and Kras (Ideno et al., 2018; Patra et al., 2018; Taki et al., 2016). We found that mice with Ga13 loss in the KPC model died faster than the control KPC mice, likely from the extensive tumor burden despite the tumors being welldifferentiated. Our findings are similar to the findings in the Kras/Gnas model, where the mice also died faster (Ideno et al., 2018; Taki et al., 2016).

We show increased E-cadherin expression at cell-cell junctions in the KPC mouse model, thus explaining the well-differentiated tumors in the KPC mice with Ga13 loss. We had previously shown that Ga13 loss results in increased E-cadherin at cell-cell junctions (Chow et al., 2016). Loss of Ga13 has also been shown to decrease endocytosis of VE-cadherin, resulting in increased VE-cadherin at cell-cell junctions (Gong et al., 2014). Earlier studies have shown that E-cadherin undergoes endocytosis following the induction of pancreatitis and subsequent recycling to regenerate cell-cell junctions and mediate acinar cell recovery (Lerch et al., 1997). Importantly, human PDAC tumors with Ga13 loss also demonstrated increased expression of E-cadherin and other cell adhesion proteins. Human PDAC tumors with 
decreased expression of Ga13 also exhibited increased activation of the mTOR signaling pathway. In agreement with the human data, we also found that Ga13 loss in the KPC mouse model enhanced mTOR signaling. Notably, GPCRs and G proteins have been shown to modulate mTOR signaling (Gan et al., 2019; Jewell et al., 2019; Nagai et al., 2020; Puertollano, 2019). For example, Gas-coupled GPCRs activate protein kinase A to inhibit mTOR complex 1 in multiple cell lines and mouse tissue (Jewell et al., 2019).

Overall, our findings increase our understanding of the role of Ga13 in vivo. We show that the phenotypic changes seen with Ga13 loss parallel many of the phenotypic changes seen with the expression of mutant Gnas (Ideno et al., 2018; Patra et al., 2018; Taki et al., 2016). However, in contrast to the previously published findings that Ga13 functions as a tumor promoter in established cell lines (Chow et al., 2016; Kelly et al., 2007; Kelly et al., 2006; Kozasa et al., 2011; Rasheed et al., 2018; Zhang et al., 2018), we demonstrate that Ga13 has a tumor-suppressive role in advanced PDAC tumors in transgenic mouse models. 


\section{METHODS}

\section{Animal experiments}

Study approval - All animal work and procedures were approved by the Northwestern University Institutional Animal Care and Use Committee. In addition, all animal experiments were performed in accordance with relevant guidelines and regulations.

Conditional knockout - Mice with loss of Ga13 in the pancreas were generated by crossing Pdx1-Cre mice (Jackson Laboratory \#014647) to mice expressing the floxed allele of Gna13 (kindly provided by Stefan Offermans, Max Planck Institute) (Moers et al., 2003), to generate $\mathrm{CGa13fl/+}$ and CGa13fl/fl mice. The bigenic mice were further crossed with mice expressing an LSL-KRas $^{\text {G12D }}$ (Jackson Laboratory \#019104) mutant allele to generate KCGa13fl/+ (KCGfl/+) or KCGa13fl/fl (KCGfl/fl) mice. These mice were further crossed with mice expressing LSL$\operatorname{Trp}^{2} 3^{\mathrm{R} 172 \mathrm{H} /+}$ (Jackson Laboratory 008652) to generate KPCGfl/+ mice. All mice were bred on a C57/BL6 background.

Cancer cell implantation - For orthotopic implantation of cancer cells, a small $(\sim 1 \mathrm{~cm})$ left abdominal side incision through skin and peritoneum of an anesthetized C57BL/6 mouse was made, and cells $\left(1 \times 10^{5}\right)$ suspended in $25 \mu \mathrm{L}$ of growth factor-reduced Matrigel (Corning Inc., Corning, NY) were injected into the tail of the pancreas using a 22-gauge Hamilton needle. The incision in the peritoneum was closed with absorbable VICRYL polyglactin sutures (Ethicon, Somerville, NJ), and the skin was closed with $7 \mathrm{~mm}$ Reflex wound clips (Roboz, Gaithersburg, MD). Tumor burden in mice was measured weekly using bioluminescence imaging using the Lago Bioluminescence system (Spectral Instrument Imaging, Tucson AZ). Mice were intraperitoneally administered D-luciferin potassium salt (GoldBio, St. Louis, MO) at a dose of $150 \mathrm{mg} / \mathrm{kg}$, and the bioluminescence signal was acquired within 10-15 minutes after injection.

For subcutaneous implantation, cancer cells $\left(1 \times 10^{5}\right)$ were suspended in $100 \mu \mathrm{L}$ of $1: 1$ mixture of PBS: Matrigel was injected under the skin in the flank of C57BL/6 mice. For shRNA induction, mice were fed water containing doxycycline $(200 \mathrm{mg} / \mathrm{L})$, which was changed every 2- 
3 days. Tumor dimensions were measured using a digital caliper, and volume was determined using the formula $V=\left(L \times W^{2}\right) / 2$, where $V$ is the volume, $L$ is length, and $W$ is the width.

Endpoint - At the experimental endpoint, mice were euthanized, pancreatic tissue weighed, and then fixed in $10 \%$ neutral buffered formalin overnight or flashed frozen for RNA or protein extraction. Fixed tissue was subsequently processed and embedded in paraffin for histological stains and immunohistochemistry.

Murine pancreatic cell lines The mouse pancreatic cell line KC4868, derived from genetically engineered Kras ${ }^{L S L-G 12 D /+}$; Pdx1-Cre (KC) C57BL/6 mice (Hingorani et al., 2005b), was provided by Dr. Paul Grippo (University of Illinois, Chicago, USA). The KC4868 cells were determined to be free of mycoplasma using MycoAlert Plus kit (Lonza Walkersville, MD) and routinely maintained in DMEM with 10\% fetal bovine serum (FBS), 1\% penicillin/streptomycin.

Cell lines expressing inducible Ga13 shRNA - KC4868 cells expressing doxycycline-inducible short hairpin RNA (shRNA) against Ga13 were generated using a lentiviral vector-based method. Four hairpins sequences against Gna13 were obtained from the RNAi Consortium database and purchased from Integrated DNA Technologies (Coralville, IA). The most potent shRNAs were found to be TRCN0000098145 and TRCN00000435189, and these were used along with control shRNA against eGFP (5'-CCGGTACAACAGCCACAACGTCTATCTCGAGATAGACGTTGTGGCTGTT GTATTTTTG-3') for subsequent experiments. Cancer cells expressing doxycycline-regulated shRNAs against Gna13 were generated using the Tet-pLKOpuro lentiviral system, deposited by Dr. Dmitri Wiederschain to Addgene (Addgene plasmid \#21915), by following their protocol (Wiederschain et al., 2009). Lentiviral particles were generated in 293T cells, and puromycin $(2 \mu \mathrm{g} / \mathrm{mL})$ was used to select transduced cells. Gene knockdown was induced by incubating cells with $1 \mu \mathrm{g} / \mathrm{mL}$ of doxycycline for 24 to 48 hours. Expression of luciferase in cancer cells was achieved by transducing cells with retroviral particles, generated in Phoenix-Eco cells, then selected using blasticidin $(10 \mu \mathrm{g} / \mathrm{mL})$. 
Generation of cell lines from transgenic mouse model - KC, KCGfl/+ or KCGfl/fl mice more than 6 months of age were euthanized and the harvested pancreatic tissue placed in $5 \mathrm{ml}$ of cold DMEM using aseptic technique. The tissue was minced using a sterile scalpel and then placed in a $2 \mathrm{mg} / \mathrm{ml}$ Collagenase I solution diluted in DMEM and enzymatically digested at $37^{\circ} \mathrm{C}$ for 30 minutes with mixing by vortex at 10-minute intervals. The digested tissue was centrifuged at 1200 rpm for 3 minutes and the cell pellet resuspended in DMEM media with $10 \%$ FBS and $1 \%$ penicillin/streptomycin. The cells were plated in a $10-\mathrm{cm}$ tissue culture dish and maintained at $37^{\circ} \mathrm{C}$ with $5 \% \mathrm{CO}_{2}$ in an incubator. The cells were serially passaged until the culture was purely cancer cells (at passage 4 or 5). For 3D matrix growth, cancer cells $\left(5 \times 10^{3}\right)$ were suspended in $500 \mu \mathrm{l}$ of Matrigel (cat \# 354234 Corning) or neutralized rat tail collagen type I (cat \# 354236 Corning) at $2 \mathrm{mg} / \mathrm{ml}$.

Immunohistochemistry For immunostains, paraffin-embedded sections were deparaffinized and rehydrated. Antigen retrieval was performed by boiling for ten minutes in sodium citrate buffer (10 mM, pH 6.0) using a pressure cooker. Endogenous peroxidase activity in tissue was quenched, and sections were blocked with a mixture of goat serum and bovine serum albumin (BSA). Tissue sections were incubated with antibodies: for Ki-67 (Cell Signaling \#12202, 1:500), cleaved caspase-3 (Cell Signaling \#9664, 1:800), MTOR (Cell Signaling \#2983, 1:500), and RPS6 (Cell Signaling \#2217, 1:1000) overnight at $4^{\circ} \mathrm{C}$. Antibody binding was detected using HRP-conjugated anti-rabbit secondary antibody and visualized using ImmPACT DAB Peroxidase Substrate kit (VectorLabs, Burlingame, CA). Photographs were taken on the FeinOptic microscope with a Jenoptik ProgRes C5 camera or TissueGnostics system and analyzed by ImageJ or HistoQuest Software.

Immunofluorescence After antigen retrieval (similar to $\mathrm{IHC}$ ), tissue sections were stained using the mouse-on-mouse (MOM) immunodetection kit (Vector Laboratories (BMK-2202). Tissue 
sections were blocked with MOM-blocking reagent for 1 hour at room temperature, followed by washing and incubation with MOM-diluent for 5 minutes. Tissue sections were then incubated with primary a primary anti-E-cadherin (BD Biosciences \#610181, 1:200) antibody for 1 hour. After washing, the sections were incubated with Alexa Fluor conjugated goat-anti mouse (AF647, \#A21235) secondary antibody for 30 minutes in the dark at room temperature. The tissue sections were washed and incubated with DAPI (Thermo Fisher D1306) for 10 minutes. Slides were mounted with fluorescence mounting media and images acquired using the EVOS M5000 microscope (Thermo Fisher).

Western blot Tissue samples were finely ground using a cold mortar and pestle. Protein was isolated from cultured cells or ground tissue using ice-cold RIPA lysis buffer containing protease and phosphatase inhibitors. The lysates were then clarified by centrifugation at $10,000 \mathrm{rpm}$ for 10 minutes at $4^{\circ} \mathrm{C}$, and the protein concentration was determined using Precision Red solution (Cytoskeleton, Inc., Denver, CO) according to the manufacturer's instructions. Equal amounts of protein were separated with a $10 \%$ SDS-PAGE electrophoresis gel. The separated proteins were transferred to a nitrocellulose membrane using the semi-dry transfer system (Bio-Rad). After blocking for one hour at room temperature with 5\% BSA, the membranes were incubated overnight at $4^{\circ} \mathrm{C}$ with primary antibodies. Primary antibodies used include Ga13 (Santa Cruz sc293424), E-cadherin (Cell Signaling 3195), Gapdh (Millipore Sigma \#MAB374), Hsp90 (Santa Cruz Biotechnology sc-7947), and alpha-tubulin (Santa Cruz Biotechnology sc-8035). HRPconjugated rabbit (A6667) or mouse (A4416) secondary antibody (Millipore-Sigma St. Louis, MO) was used with SuperSignal West Pico PLUS (Thermo Fisher Scientific) for protein detection.

$\boldsymbol{q R T}-\boldsymbol{P C R}$ RNA was isolated from cultured cells or tissue using the RNeasy kit (74104, QIAGEN, Hilden, Germany) according to the manufacturer's instructions. cDNA was 
synthesized from 1-2 $\mu \mathrm{g}$ of RNA with random hexamers and M-MLV reverse transcriptase in a single $50 \mu \mathrm{L}$ reaction. Quantitative PCR was performed using gene-specific TaqMan probes, TaqMan Universal PCR Master Mix, and the CFX Connect Real-Time PCR Detection System (Bio-Rad Hercules, CA). Probes used to detect the following genes: Gna13 (Mm01250415_m1), Gna11 (Mm01172792_m1), Gna12 (Mm00494665_m1), Gnaq (Mm00492381_m1), Gnas (Mm01242435_m1). Mouse Gapdh (Mm99999915_g1) and Rn18s (Mm03928990_g1) were used as endogenous controls, and their expression levels were used to calculate the relative expression using the comparative $C_{t}$ method (Livak and Schmittgen, 2001).

TCGA data analysis Expression data from RNA and protein (RPPA) analyses for human pancreatic ductal adenocarcinoma patients in The Cancer Genome Atlas (TCGA) were extracted from cBioportal (Cerami et al., 2012; Gao et al., 2013). Patient IDs from the study are listed in Table 1 and are categorized as high (GNA13 hi, upper 25\%, n= 43) or low (GNA13 lo, lower $25 \%, n=43$ ) expression of GNA13 RNA.

Statistics The in vivo and in vitro results were compared using one-way ANOVA and 2-tailed $t$ test analysis. Error bars represent the standard error of the mean or standard deviation as specified in the figure legends. All statistical analyses were done using GraphPad Prism. A pvalue of less than 0.05 was considered significant. 


\section{ACKNOWLEDGEMENTS}

We thank Dr. Stefan Offermanns (Max-Planck-Institute for Heart and Lung Research, Bad Nauheim, Germany), who kindly provided the Gna $13^{\text {floxfllox }}$ mice. This work was supported by grants R01CA217907 (to H.G.M.), R21CA255291 (to H.G.M.), a Merit award I01BX002922 (to H.G.M.) from the Department of Veterans Affairs, APA/APA Foundation 2020 Young Investigator Pancreatitis Grant (to M.A.S.), the Mander Foundation Award (to H.G.M.) and the Harold E. Eisenberg Foundation Award (to T.N.D.P.) from the Robert H. Lurie Cancer Center, and the $\mathrm{NIH} / \mathrm{NCl}$ training grant T32 CA070085 (to T.N.D.P).

AUTHOR CONTRIBUTIONS M.A.S. designed the studies, performed the experiments, analyzed the data, and wrote the manuscript. C.S., M.G.K., and T.N.D.P. performed the experiments and analyzed the data. H.G.M. designed the studies, analyzed the data, wrote the manuscript, and secured funding. All authors edited and approved the final manuscript. 


\section{REFERENCES}

Cerami, E., Gao, J., Dogrusoz, U., Gross, B.E., Sumer, S.O., Aksoy, B.A., Jacobsen, A., Byrne, C.J., Heuer, M.L., Larsson, E., et al. (2012). The cBio cancer genomics portal: an open platform for exploring multidimensional cancer genomics data. Cancer Discov 2, 401-404.

Chow, C.R., Ebine, K., Knab, L.M., Bentrem, D.J., Kumar, K., and Munshi, H.G. (2016). Cancer Cell Invasion in Three-dimensional Collagen Is Regulated Differentially by Galpha13 Protein and Discoidin Domain Receptor 1-Par3 Protein Signaling. J Biol Chem 291, 1605-1618.

Farrell, J.J., and Fernandez-del Castillo, C. (2013). Pancreatic cystic neoplasms: management and unanswered questions. Gastroenterology 144, 1303-1315.

Gan, L., Seki, A., Shen, K., Iyer, H., Han, K., Hayer, A., Wollman, R., Ge, X., Lin, J.R., Dey, G., et al. (2019). The lysosomal GPCR-like protein GPR137B regulates Rag and mTORC1 localization and activity. Nat Cell Biol 21, 614-626.

Gao, J., Aksoy, B.A., Dogrusoz, U., Dresdner, G., Gross, B., Sumer, S.O., Sun, Y., Jacobsen, A., Sinha, R., Larsson, E., et al. (2013). Integrative analysis of complex cancer genomics and clinical profiles using the cBioPortal. Sci Signal 6, pl1.

Gong, H., Gao, X., Feng, S., Siddiqui, M.R., Garcia, A., Bonini, M.G., Komarova, Y., Vogel, S.M., Mehta, D., and Malik, A.B. (2014). Evidence of a common mechanism of disassembly of adherens junctions through Galpha13 targeting of VE-cadherin. J Exp Med 211, 579-591.

Hauser, A.S., Attwood, M.M., Rask-Andersen, M., Schioth, H.B., and Gloriam, D.E. (2017). Trends in GPCR drug discovery: new agents, targets and indications. Nat Rev Drug Discov 16, 829-842.

Healy, J.A., Nugent, A., Rempel, R.E., Moffitt, A.B., Davis, N.S., Jiang, X., Shingleton, J.R., Zhang, J., Love, C., Datta, J., et al. (2016). GNA13 loss in germinal center B cells leads to impaired apoptosis and promotes lymphoma in vivo. Blood 127, 2723-2731.

Hingorani, S.R., Petricoin, E.F., Maitra, A., Rajapakse, V., King, C., Jacobetz, M.A., Ross, S., Conrads, T.P., Veenstra, T.D., Hitt, B.A., et al. (2003). Preinvasive and invasive ductal pancreatic cancer and its early detection in the mouse. Cancer Cell 4, 437-450.

Hingorani, S.R., Wang, L., Multani, A.S., Combs, C., Deramaudt, T.B., Hruban, R.H., Rustgi, A.K., Chang, S., and Tuveson, D.A. (2005a). Trp53R172H and KrasG12D cooperate to promote chromosomal instability and widely metastatic pancreatic ductal adenocarcinoma in mice. Cancer Cell 7, 469-483.

Hingorani, S.R., Wang, L., Multani, A.S., Combs, C., Deramaudt, T.B., Hruban, R.H., Rustgi, A.K., Chang, S., and Tuveson, D.A. (2005b). Trp53R172H and KrasG12D cooperate to promote chromosomal instability and widely metastatic pancreatic ductal adenocarcinoma in mice. Cancer Cell 7, 469-483.

Ideno, N., Yamaguchi, H., Ghosh, B., Gupta, S., Okumura, T., Steffen, D.J., Fisher, C.G., Wood, L.D., Singhi, A.D., Nakamura, M., et al. (2018). GNAS(R201C) Induces Pancreatic Cystic 
Neoplasms in Mice That Express Activated KRAS by Inhibiting YAP1 Signaling. Gastroenterology 155, 1593-1607 e1512.

Jewell, J.L., Fu, V., Hong, A.W., Yu, F.X., Meng, D., Melick, C.H., Wang, H., Lam, W.M., Yuan, H.X., Taylor, S.S., et al. (2019). GPCR signaling inhibits mTORC1 via PKA phosphorylation of Raptor. Elife 8.

Kelly, P., Casey, P.J., and Meigs, T.E. (2007). Biologic functions of the G12 subfamily of heterotrimeric g proteins: growth, migration, and metastasis. Biochemistry 46, 6677-6687.

Kelly, P., Moeller, B.J., Juneja, J., Booden, M.A., Der, C.J., Daaka, Y., Dewhirst, M.W., Fields, T.A., and Casey, P.J. (2006). The G12 family of heterotrimeric G proteins promotes breast cancer invasion and metastasis. Proceedings of the National Academy of Sciences 103, 81738178.

Kozasa, T., Hajicek, N., Chow, C.R., and Suzuki, N. (2011). Signalling mechanisms of RhoGTPase regulation by the heterotrimeric $\mathrm{G}$ proteins $\mathrm{G} 12$ and $\mathrm{G} 13$. Journal of Biochemistry 150, 357-369.

Kurose, H. (2003). Galpha12 and Galpha13 as key regulatory mediator in signal transduction. Life Sci 74, 155-161.

Lerch, M.M., Lutz, M.P., Weidenbach, H., Muller-Pillasch, F., Gress, T.M., Leser, J., and Adler, G. (1997). Dissociation and reassembly of adherens junctions during experimental acute pancreatitis. Gastroenterology 113, 1355-1366.

Livak, K.J., and Schmittgen, T.D. (2001). Analysis of relative gene expression data using realtime quantitative PCR and the 2(-Delta Delta C(T)) Method. Methods 25, 402-408.

Matthaei, H., Schulick, R.D., Hruban, R.H., and Maitra, A. (2011). Cystic precursors to invasive pancreatic cancer. Nat Rev Gastroenterol Hepatol 8, 141-150.

Moers, A., Nieswandt, B., Massberg, S., Wettschureck, N., Gruner, S., Konrad, I., Schulte, V., Aktas, B., Gratacap, M.P., Simon, M.I., et al. (2003). G13 is an essential mediator of platelet activation in hemostasis and thrombosis. Nat Med 9, 1418-1422.

Molin, M.D., Matthaei, H., Wu, J., Blackford, A., Debeljak, M., Rezaee, N., Wolfgang, C.L., Butturini, G., Salvia, R., Bassi, C., et al. (2013). Clinicopathological correlates of activating GNAS mutations in intraductal papillary mucinous neoplasm (IPMN) of the pancreas. Ann Surg Oncol 20, 3802-3808.

Muppidi, J.R., Schmitz, R., Green, J.A., Xiao, W., Larsen, A.B., Braun, S.E., An, J., Xu, Y., Rosenwald, A., Ott, G., et al. (2014). Loss of signalling via Galpha13 in germinal centre B-cellderived lymphoma. Nature 516, 254-258.

Nagai, M.H., Xavier, V.P.S., Gutiyama, L.M., Machado, C.F., Reis, A.H., Donnard, E.R., Galante, P.A.F., Abreu, J.G., Festuccia, W.T., and Malnic, B. (2020). Depletion of Ric-8B leads to reduced mTORC2 activity. PLoS Genet 16, e1008255.

O'Hayre, M., Inoue, A., Kufareva, I., Wang, Z., Mikelis, C.M., Drummond, R.A., Avino, S., Finkel, K., Kalim, K.W., DiPasquale, G., et al. (2016). Inactivating mutations in GNA13 and RHOA in 
Burkitt's lymphoma and diffuse large B-cell lymphoma: a tumor suppressor function for the Galpha13/RhoA axis in B cells. Oncogene 35, 3771-3780.

Oldham, W.M., and Hamm, H.E. (2008). Heterotrimeric G protein activation by G-proteincoupled receptors. Nat Rev Mol Cell Biol 9, 60-71.

Patra, K.C., Kato, Y., Mizukami, Y., Widholz, S., Boukhali, M., Revenco, I., Grossman, E.A., Ji, F., Sadreyev, R.I., Liss, A.S., et al. (2018). Mutant GNAS drives pancreatic tumourigenesis by inducing PKA-mediated SIK suppression and reprogramming lipid metabolism. Nat Cell Biol 20, 811-822.

Pham, T.N.D., Shields, M.A., Spaulding, C., Principe, D.R., Li, B., Underwood, P.W., Trevino, J.G., Bentrem, D.J., and Munshi, H.G. (2021). Preclinical Models of Pancreatic Ductal Adenocarcinoma and Their Utility in Immunotherapy Studies. Cancers (Basel) 13.

Puertollano, R. (2019). GPCRs join the mTORC1 regulatory network. Nat Cell Biol 21, 538-539. Rasheed, S.A.K., Leong, H.S., Lakshmanan, M., Raju, A., Dadlani, D., Chong, F.T., Shannon, N.B., Rajarethinam, R., Skanthakumar, T., Tan, E.Y., et al. (2018). GNA13 expression promotes drug resistance and tumor-initiating phenotypes in squamous cell cancers. Oncogene 37, 13401353.

Sriram, K., Salmeron, C.S., Wiley, S.Z., and Insel, P.A. (2020). GPCRs in pancreatic adenocarcinoma: Contributors to tumor biology and novel therapeutic targets. Br J Pharmacol. Syrovatkina, V., Alegre, K.O., Dey, R., and Huang, X.Y. (2016). Regulation, Signaling, and Physiological Functions of G-Proteins. J Mol Biol 428, 3850-3868.

Syrovatkina, V., and Huang, X.Y. (2019). Signaling mechanisms and physiological functions of G-protein Galpha13 in blood vessel formation, bone homeostasis, and cancer. Protein Sci 28, 305-312.

Taki, K., Ohmuraya, M., Tanji, E., Komatsu, H., Hashimoto, D., Semba, K., Araki, K., Kawaguchi, Y., Baba, H., and Furukawa, T. (2016). GNAS(R201H) and Kras(G12D) cooperate to promote murine pancreatic tumorigenesis recapitulating human intraductal papillary mucinous neoplasm. Oncogene 35, 2407-2412.

Tutunea-Fatan, E., Lee, J.C., Denker, B.M., and Gunaratnam, L. (2020). Heterotrimeric Galpha12/13 proteins in kidney injury and disease. Am J Physiol Renal Physiol 318, F660-F672. Wiederschain, D., Wee, S., Chen, L., Loo, A., Yang, G., Huang, A., Chen, Y., Caponigro, G., Yao, Y.M., Lengauer, C., et al. (2009). Single-vector inducible lentiviral RNAi system for oncology target validation. Cell Cycle 8, 498-504.

Wu, J., Matthaei, H., Maitra, A., Dal Molin, M., Wood, L.D., Eshleman, J.R., Goggins, M., Canto, M.I., Schulick, R.D., Edil, B.H., et al. (2011). Recurrent GNAS mutations define an unexpected pathway for pancreatic cyst development. Sci Transl Med 3, 92ra66.

Zhang, Z., Tan, X., Luo, J., Cui, B., Lei, S., Si, Z., Shen, L., and Yao, H. (2018). GNA13 promotes tumor growth and angiogenesis by upregulating CXC chemokines via the NF-kappaB signaling pathway in colorectal cancer cells. Cancer Med 7, 5611-5620. 


\section{FIGURE LEGENDS}

Figure 1: Ga13 loss does not affect tumor development in the $\mathrm{KC}$ transgenic mouse model. A, Alleles (left) of KrasG12D (K), Pdx1-Cre (C), and Ga13fl (G) and Western blot showing Ga13 expression levels in the pancreas of mice of indicated genotypes. Hsp90 was used as an endogenous control. B, Expression of Gna13, Gna11, Gna12, Gnaq, and Gnas was determined by qRT-PCR. Gapdh was used as an endogenous control. t-test $(n=3,3),{ }^{* \star *} p$ value $\leq 0.001$, Mean $\pm S D$. C, Representative pictures from H\&E stains of the pancreas from $\mathrm{KCG}+/+, \mathrm{KCGfl} /+$, and $\mathrm{KCGfl} / \mathrm{fl}$ mice at 3 and 6 months. The whole profile of pancreas tissue was scanned, and the percentage of pancreas with lesions was analyzed at 3 and 6 months (n $=7,8,7$ at 3 months; $n=8,11,6$ at 6 months). t-test Mean $\pm S D$. Scale bar $=100 \mu m$. D, Immunostains and quantification of $\mathrm{Ki} 67$ in the $\mathrm{KCG}+/+, \mathrm{KCGfl} /+$, and $\mathrm{KCGfl} / \mathrm{fl}$ mice at 6 months. t-test, mean \pm SD. Scale bar $=100 \mu \mathrm{m}$. E, Kaplan-Meier survival analysis of $\mathrm{KCG}+/+$ $(n=14), K C G f l /+(n=26)$, and KCGfl/fl $(n=15)$ mice.

Figure 2: Cell lines established from KC mice with Ga13 loss grow slower in vitro and in vivo. A, Western blot for Ga13 (short and long exposure) in pancreatic cancer cell lines established from KCG+/+ (2005), KCGfl/+ (1548), and KCGfl/fl (1930) mice. B, Equal numbers $\left(5 \times 10^{3}\right)$ of $\mathrm{KCG}+/+(2005), \mathrm{KCGfl} /+(1548)$, and $\mathrm{KCGfl} / \mathrm{fl}(1930)$ cells were plated in 6-well tissue culture plates in duplicate, and proliferation was determined by counting daily the number of cells present. This experiment was repeated at least three times. C, Representative picture of KCG+/+ (2005), KCGfl/+ (1548), and KCGfl/fl (1930) cells grown in 3D type I collagen (top) and Matrigel (bottom) cultures after 5 days. Scale bar $=100 \mu \mathrm{m}$. D,E, KCG+/+ (2005), KCGfl/+ (1548), and KCGfl/fl (1930) cells were implanted subcutaneously in the flank of female B6 mice (6-8 weeks old, $n=5$; two tumors per mouse). The tumor sizes were measured using a caliper $(\boldsymbol{D})$, harvested $\sim$ day 50, photographed, and wet-weight was measured $(\boldsymbol{E})$. One-way ANOVA mean $\pm S D{ }^{*} p$-value $\leq 0.05,{ }^{* *} p$-value $\leq 0.01,{ }^{* * *} p$-value $\leq 0.001 ;{ }^{* * *} p$-value $\leq 0.0001$. 
Figure 3: KPC mice with Ga13 loss develop differentiated tumors with increased Ecadherin expression. A, Alleles of KrasG12D (K), Pdx1-Cre (C), Ga13fl (G), and Trp53R172H/+ (P). B, Ga13 expression levels in the pancreas of mice of indicated genotypes were determined by Western blotting. Hsp90 was used as an endogenous control. Expression of Gna13, Gna11, Gna12, Gnaq, and Gnas was determined by qRT-PCR. Gapdh was used as an endogenous control. Unpaired t-test $(n=3,3)$, ${ }^{* *} p$-value $\leq 0.01$. Mean $\pm S D$. C, Representative pictures (left) from H\&E stains of the pancreas from KPCG+/+ and KPCGfl/+ mice at 3 months. The whole profile of pancreas tissue was scanned, and the relative lesion burden and the lesion type were quantified at 3 months $(n=9,9)$. t-test Mean $\pm S D$. Scale bar $=$ $100 \mu \mathrm{m} . \mathbf{D}, \mathrm{H} \& \mathrm{E}$ and immunofluorescence stains for E-cadherin in the pancreatic tissue from $\mathrm{KPCG}+/+$ and $\mathrm{KPCGfl} /+$ mice at the endpoint. The relative fluorescence intensity at cell-cell junctions in the lesions was quantified using ImageJ. E, Immunohistochemical stains for Ecadherin in the pancreatic tissue from $\mathrm{KPCG}+/+$ and $\mathrm{KPCGfl} /+$ mice at the endpoint. The relative E-cadherin score at cell-cell junctions in the lesions was quantified using HistoQuest Software. F, Immunostains and quantification of $\mathrm{Ki} 67$ in the $\mathrm{KPCG}+/+$ and $\mathrm{KPCGfl} /+$ mice $(\mathrm{n}=8$, 7). t-test, mean $\pm S D$. Scale bar $=100 \mu \mathrm{m}$.

Figure 4: KPCGfl/+ mice demonstrate increased mTOR signaling and decreased survival.

A, Analysis of samples in cBioportal with low and high expression of GNA13 for E-cadherin (CDH1), $\beta$-catenin (CTNNB1), and Claudin-7 (CLDN7) expression at the protein (RPPA) and gene expression (RSEM) levels $(n=43,43)$. t-test, mean \pm SEM. B, Analysis of samples in cBioportal with low and high expression of GNA13 for RPS6, PDK1 (p-S241), EEF2, and MTOR at the protein (RPPA) levels. Effects on RPS6 and MTOR were also analyzed at the gene expression (RSEM) levels. t-test, mean \pm SEM ${ }^{*}, p$-value $\leq 0.05 ;{ }^{* *}, p$-value $\leq 0.01 . \mathbf{C}$, 
Immunostains and quantification for $\operatorname{Rps} 6(n=6,6)$ and Mtor $(n=7,7)$ in advanced tumors developing in $\mathrm{KPCG}+/+$ and $\mathrm{KPCGfl} /+$ mice. t-test, mean \pm SD. Scale bar $=100 \mu \mathrm{m}$. D, KaplanMeier survival analysis of KPCG+/+ $(n=21)$ and KPCGfl/+ $(n=28)$ mice using long-rank test. 


\section{SUPPLEMENTAL INFORMATION}

\section{Supplemental Figure S1: Ga13 shRNA in KC cell lines decreases tumor growth in vivo. A,} $4868 \mathrm{KC}$ cells expressing doxycycline-inducible short hairpin RNA against GFP or Ga13 were treated with doxycycline $(1 \mu \mathrm{g} / \mathrm{ml})$ and analyzed by western blot (top, $48 \mathrm{~h}$ ) and qRT-PCR (bottom, 24h). a-tubulin and Gapdh were used as an endogenous control for western blot and qRT-PCR, respectively. The experiment was conducted at least three times. t-test, Mean $\pm S D$. ${ }^{* *}, p<0.01 ;{ }^{* *}, p<0.001 . B, 4868 \mathrm{KC}$ cells $\left(1 \times 10^{4}\right)$ expressing shGFP or shRNA against Ga13 (sh45 or sh89) were implanted subcutaneously in the flank of female mice (6-8 weeks old). After

2 weeks, mice were fed doxycycline containing water $(200 \mathrm{mg} / \mathrm{L})$. The tumor sizes were measured using a caliper until an endpoint was reached, then harvested and stained using H\&E. One-way ANOVA ( $n=3,3,2$; two tumors per mouse). Mean $\pm S D$. Scale bar $=1 \mathrm{~mm}$. C, $4868 \mathrm{KC}$ cells $\left(1 \times 10^{4}\right)$ expressing shGFP or shRNA against Ga13 (sh45) and co-expressing luciferase were implanted in the pancreas of mice at 8 weeks of age. Tumor burden was measured weekly using bioluminescence imaging. Shown here are images after 35 days. At the endpoint, tumors were harvested, wet-weight measured, and then stained using $H \& E$. $(n=3$, 3) t-test Mean $\pm S D$. Scale bar $=1 \mathrm{~mm}$.

SUPPLEMENTAL TABLE S1: Expression data from RNA and protein (RPPA) analyses for human pancreatic ductal adenocarcinoma patients in The Cancer Genome Atlas (TCGA) were extracted from cBioportal. Expression of GNA13 RNA is categorized as high (GNA13 hi, upper $25 \%, n=43$ ) or low (GNA13 lo, lower $25 \%, n=43$ ). Table 1 lists patient IDs from TCGA used in our analysis. 
A

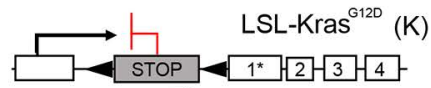

\begin{tabular}{|l|l|}
\hline Pdx-1 & Cre \\
\hline
\end{tabular}

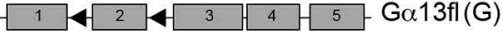
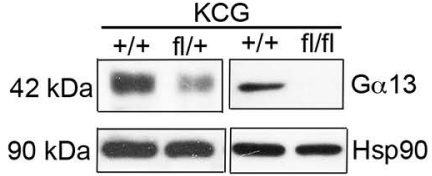

C

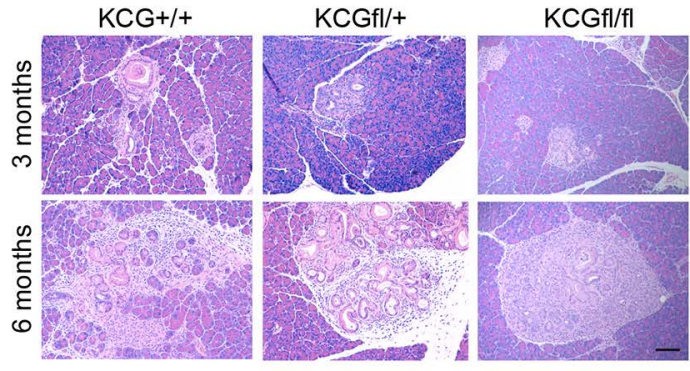

$H \& E$

D

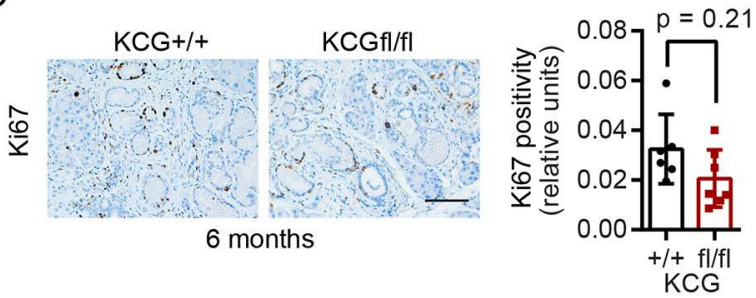

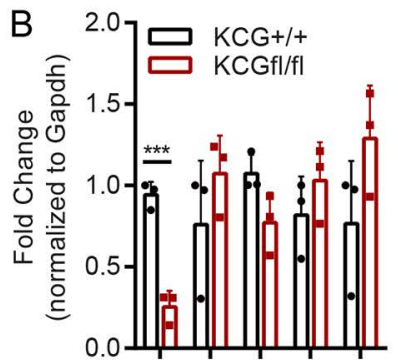

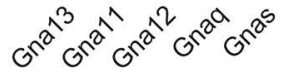

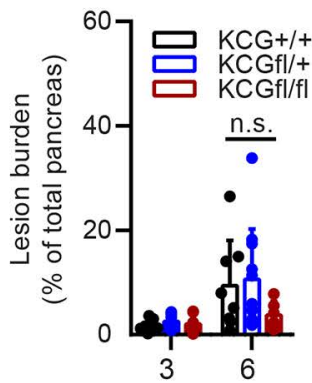

Time/months

$E$

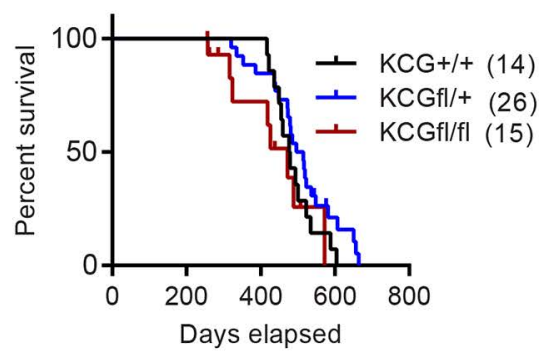


Figure 2

A
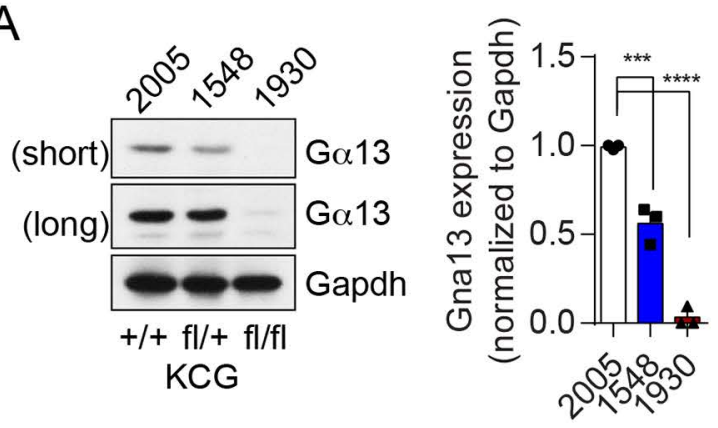

c

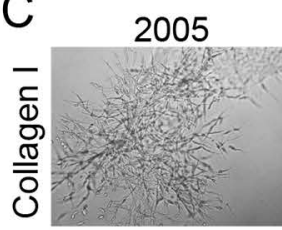

1548

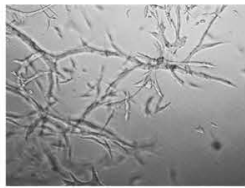

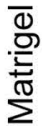

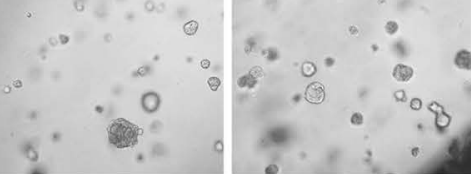

E

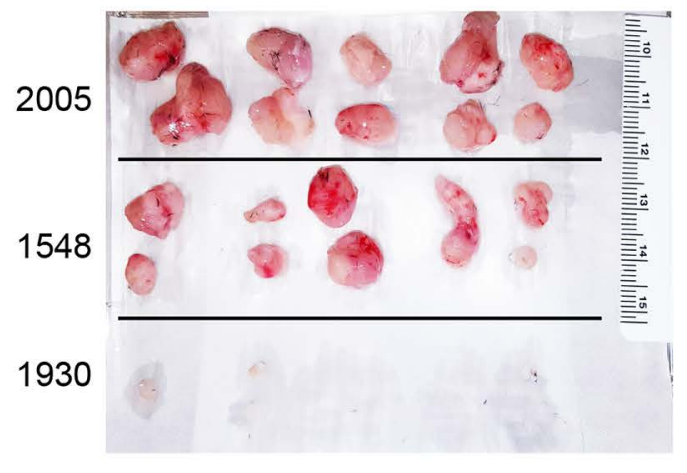

B
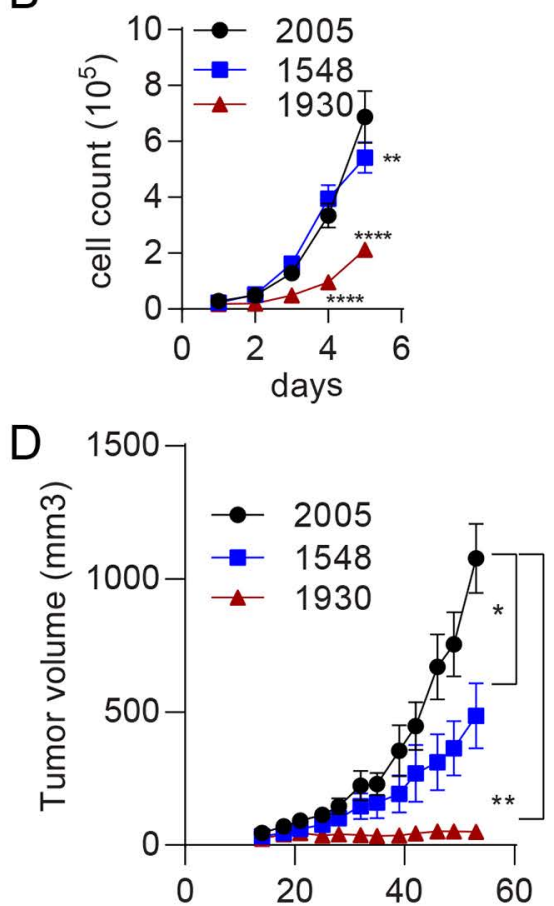

Time after transplant

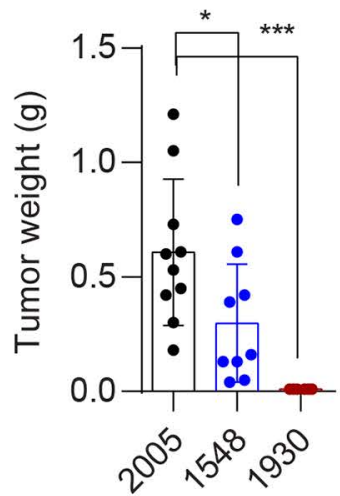


Figure 3

A

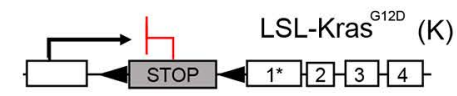

-1 STOP-2-13-4 $45^{*}-\mathrm{p} 53^{\mathrm{R} 172 \mathrm{H}}(\mathrm{P})$

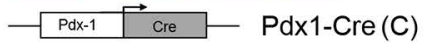

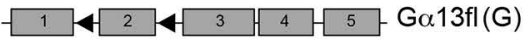

C

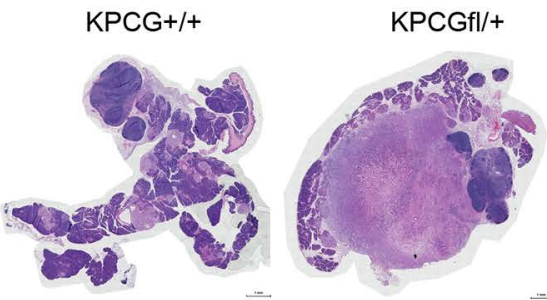

D

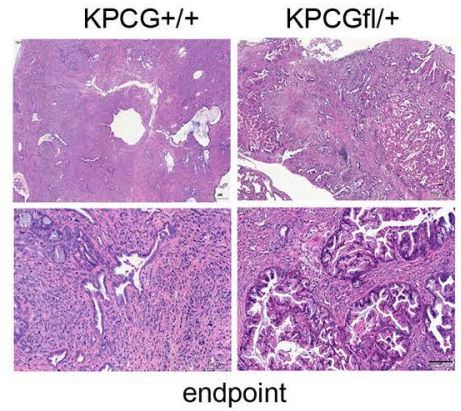

E E-cadherin IHC

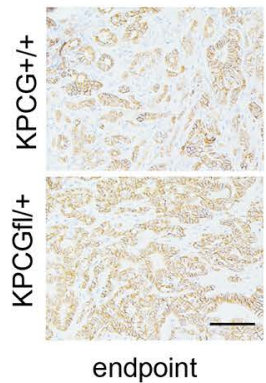

endpoint
B

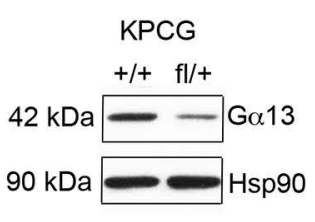

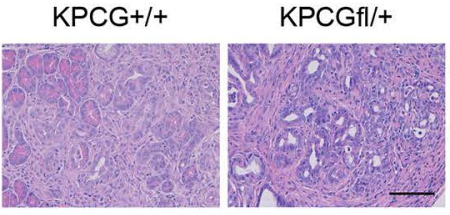

3 months

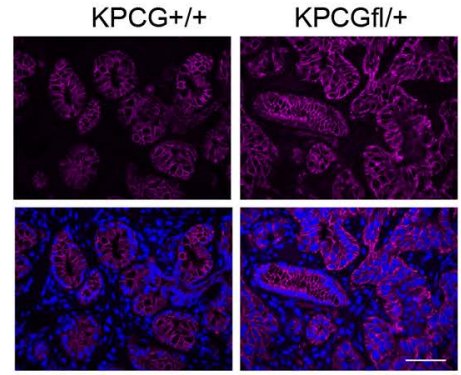

endpoint
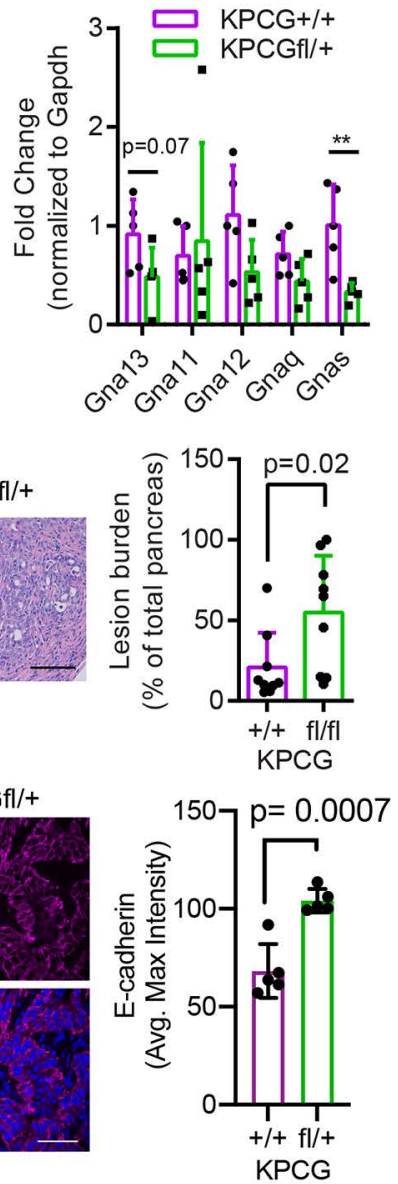

F

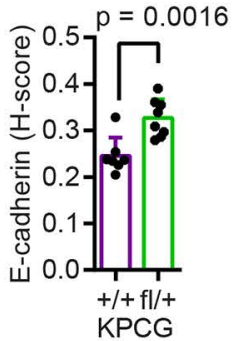

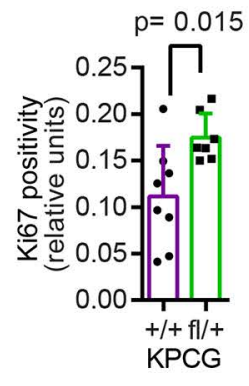


A

Figure 4

Human PDAC
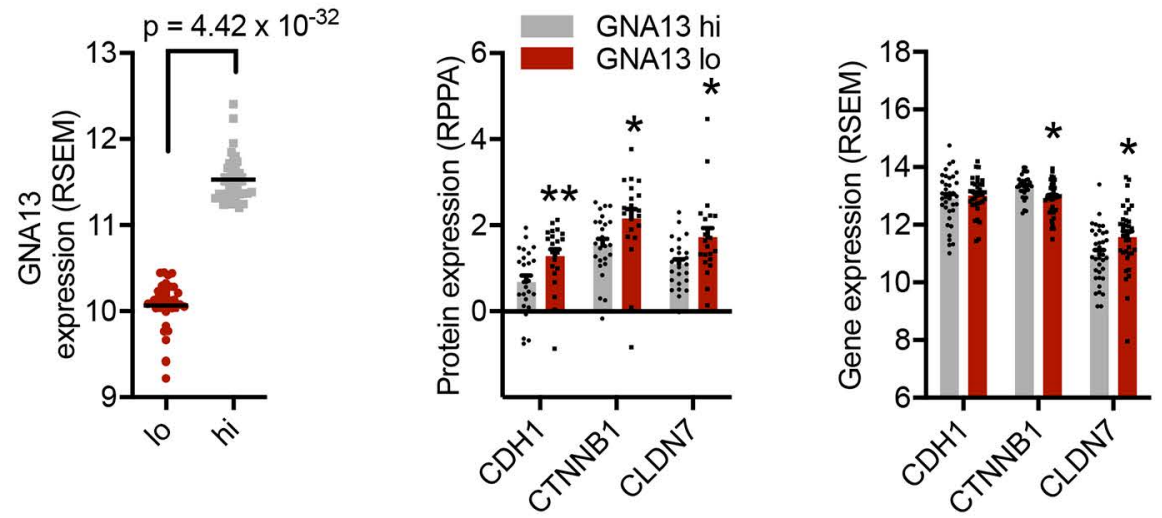

B

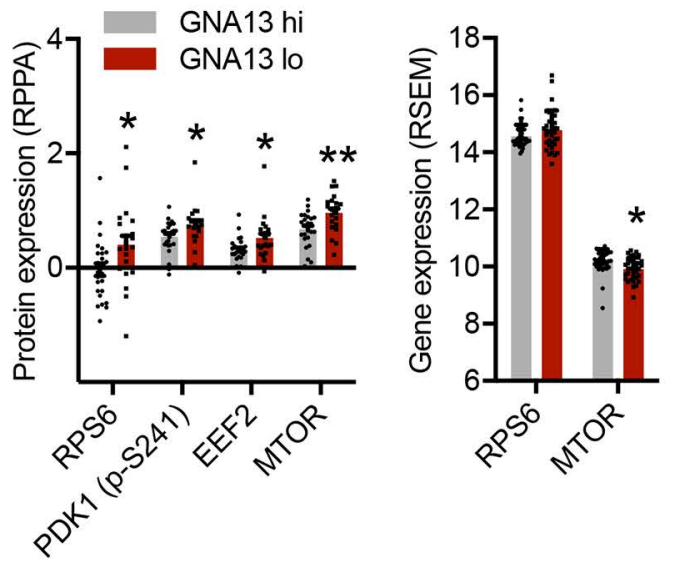

D

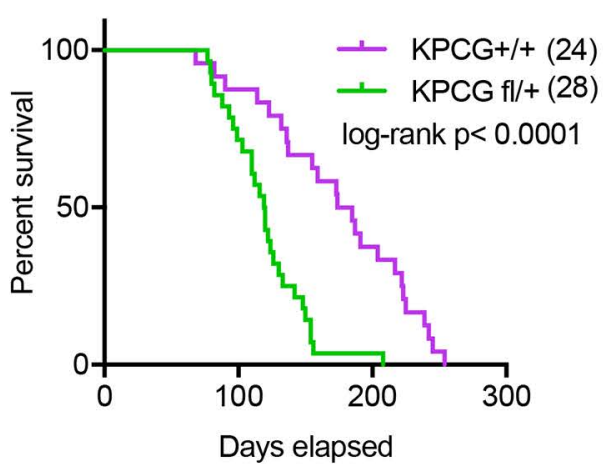

c
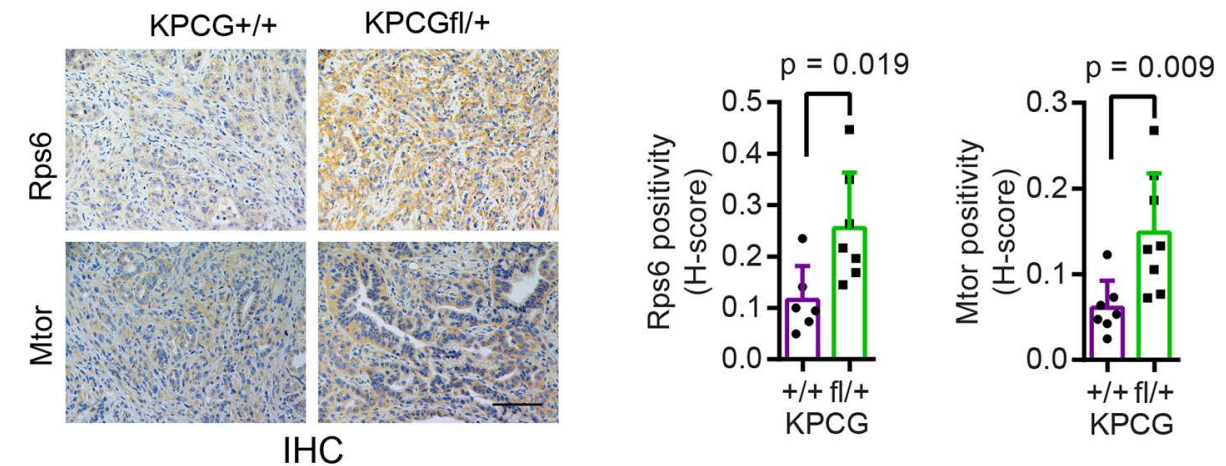